\title{
Index
}

Aeschylus 150, 153

Agamemnon 231-232

Danaids 199-200

Eumenides 22-23

Mysians 55

Seven Against Thebes 217-234

parodos 220-224

$563-566 \quad 230-231$

Suppliants 231-232

Telephus 55

Aesop 118-119, 123-125, 130, 142

Agathon 6

Alcaeus 14, 66, 153, 175, 192, 322

fr. $129115 \mathrm{n} 31$

fr. $140 \quad 204-207,215$

fr. $141 \quad 153$

fr. $249 \quad 166$

fr. 345153,167

fr. $348 \quad 167$

Alcman 13, 195, 271, 363, 343, 347

PMGF

$1 \quad 3,76-77,96,198$ n $35,219-220,257-$ $260,268,272,302$

3 260-262, 297-298, 363n 43

$26 \quad 323-324,365 n 48$

Alexandrian scholarship $\quad 53,61,66,152,292$, 308-309

Anacreon 13, 302, 359, 363, 364, 366, 376, 382

$P M G$

$$
\begin{array}{ll}
413 & 302 \\
459 & 299
\end{array}
$$

Anacreontea 13, 359, 364, 372

$$
\begin{array}{lr}
16 & 370-384 \\
17 & 370-384 \\
53.1-8 & 364
\end{array}
$$

Antiphanes 161

Apollodorus $\quad 5^{2-53}$

Archilochus 6o, 63-64

fr. 1053

fr. $118 \quad 188$

fr. $172 \quad 125$

frr. $185^{-187} \quad 108-142$

fr. $196 \quad 300-301$

'Telephus elegy' 12, 56-58, 60, 66

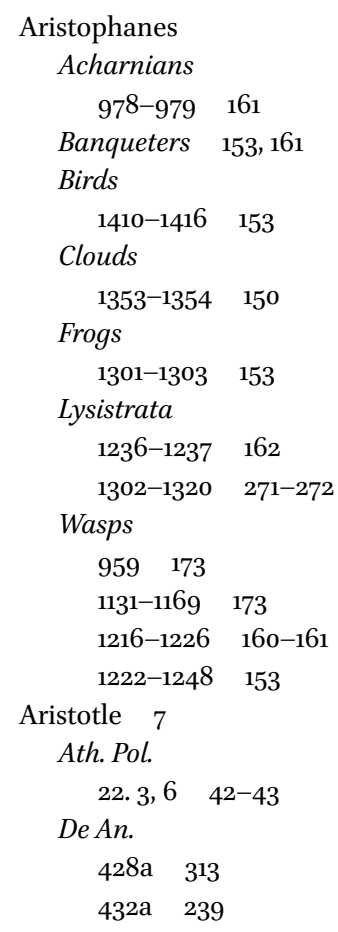

Hist. An.

502a-b 113

Pol.

$1342 \mathrm{~b} \quad 170$

$P M G$

$842 \quad 162-163$

Aristoxenus 154, 157

fr. 99159

fr. $125 \quad 148-149$

Asclepiades

Epigram 3 (Sens) 197n3o

Athenaeus 153-168, 193-194, 206, $362 n 42$

Aulus Gellius 344, 363, 366

Attic Nights

19.9.4 359

Bacchylides $\quad 17$

Ode

$3.17-22 \quad 19$

$5.176-186 \quad 264-265$

$13 \quad 269$ 


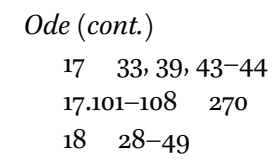

Barthes, Roland $321,330,376$

Brygos painter $35^{-} 36,176$ n 70

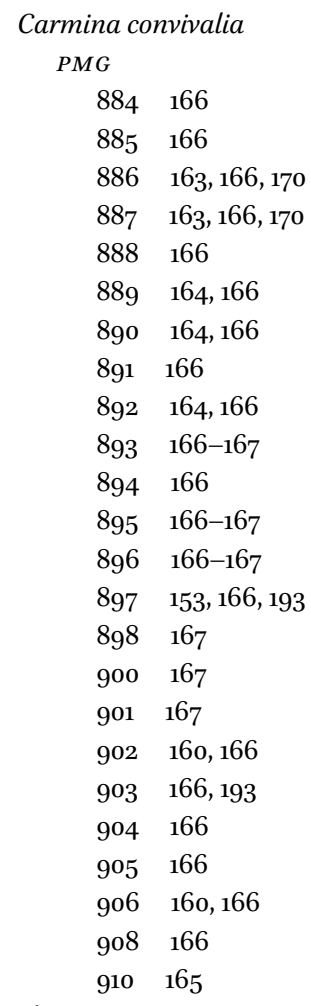

choreia $322,324,358,361,364-366$

chorality $2,69,312,314,343,366$

Cimon 17, 37-45

cultural memory $\quad 312$

Cypria $\quad 5^{1-53,55}$

fr. 2053

dance $2,11,118 \mathrm{n} 42,163,240 \mathrm{n} 12,243,248,260$, 263, 266, 269-282, 291, 298, 311-328, 335, $358,372 \mathrm{n} 3$

Bacchic $105 \mathrm{n} 48$

choral $\quad 69-100,218-219,225,226 \mathrm{n} 35,312$, $314-315,318,322,345-347,360,360-$ 366

sympotic 11-12, 109, 121-141

deixis 11, 240, 249, 268, 295, 313
Derveni Papyrus $\quad$ 290, 329
Dickinson, Emily 1
Dicaearchus $\quad 148-153$

ecphrasis 10, 78, 242-246, 248, 312, 352, 355$358,373,383$

ecphrastic discourse 10

Empedocles 100, 288-290

enargeia $8,240,243,245^{-246,248, ~ 312, ~}$ $335 \mathrm{n} 16,356 \mathrm{n} 18,376$

Eos $\quad 321-322,324,328,330$

epic $9,51,66,69,103,297,312$

Euphranor 8

Euripides 6

Andromache 20, 22

Electra 275

parodos 273

Helen

1313-1313 278

Ion 21-22, 45

Iphigenia in Tauris

1143-t2 274

Trojan Women 338-339 274

Eusebius $\quad 191$

gaze $13,185,246,248,256,259,275$, $282,373-374,381$

choral $74-75,96$

erotic 290-294, 299-300, 303-

304

Helios 245, 319, 324, 329

Hephaestion 186, 194-196, 201

Heraclitus 'the Allegorist' 3

Heraclitus of Ephesus $\quad$ 228, 329

Hermogenes $350,352,366$

Herodotus 66, 228

6.126-130 122

Hesiod 51, 102, 112

Catalogue of Women $\quad 54-55$

Theogony 300

1080

120-121 300-301

Hipponax $113,115 \mathrm{n} 26,165 \mathrm{n} 36$

fr. $119 \mathrm{~W}^{2} \quad 188,295$

Homer $71,73,289,332,360$

Iliad

$2.484-486 \quad 228$ 


$$
\begin{array}{lc}
14.182-183 & 99 \mathrm{n} 43 \\
14.292-296 & 301 \\
16.181-185 & 277 \\
18.478-608 & 70 \\
21.220 & 58
\end{array}
$$

Odyssey

6.99-109 279-280

6.153-159 280-281

8.248-249 121

$8.260-278 \quad 81$

$8.489 \quad 376$

$8.491 \quad 376$

12.3-3 321

$18.29899 n 43$

Homeric Hymns

to Apollo 80

149-155 74-75

156-176 102

to Aphrodite

117-120 277-278

119-120 79

221-224 320

to Demeter 4-8 $\quad 278$

Horace 7

Ibycus 302

PMGF

$287 \quad 299$

initiation $198-201,255^{-283}, 291-296$

ivory $32,353-355,370-371,375$

kinaesthesia $\quad 311,313$

Lacan, Jacques $\quad 377 \mathrm{n} 24,379$

lament $\quad 9-10,166,169,314,323,33^{2-3} 33$

lyric 'I' 265n31, 318, 320-321, 324, 327, 346

Lucian

Piscator

$36 \quad 118$

Macedonia $\quad 5^{-6}$

Melanippides 6

Menander Rhetor 363,366

metaphor $3,77,86,179-180,204,232,238-$ 250, 257, 289-295, 299, 303 313, 324, 355, 375

mimesis 44, 73-74, 77, 82, 102-104, 109, 141, $219,25^{\circ}, 35^{6}-35^{8}, 377,384$
Mimnermus

fr. $4 \mathrm{~W} \quad 325$

Muses $80,101,103,115,266,303315^{-316}$, 316 n $35,318,320,323,335,376$

naturalistic representation $\quad 60,90,373,377^{-}$ 378,383

New Music 5

Orpheus / Orphism 290, 314, 322, 328-329, $331,334-335$

Palatine Anthology $\quad 344,359-361,374 n 12,379$ 9.189360

painting $1,4,6-11,39-40,142,35^{2-}-35^{8}, 370-$ 384

Pausanias 51

$1.15 \cdot 3 \quad 39-40$

$1.17 \cdot 2-3 \quad 39$

3.26.9-10 63n27

10.11.5 23

$10.25-31 \quad 9 n 21$

performance $4-5,308-310,313,315,322$, 346

choral $349,35^{8-359,360,362,366}$

Hellenistic 308

solo 2

sympotic (re)performance 192, 331, 372 phantasia 240-241, 247-249, 313, 373, 376, 38on 34,382

Phaon $321,327,329,332$

Philitas of Cos 56

Philodemus

Epigram 14.2 Sider $\quad 187$

De mus. $4.12 \quad 158$

Philostratus $4,12,51,58-59,60,361,363$, 366

Heroicus

$$
\begin{array}{lll}
2.15 & 60 \\
23.24-25 & 5^{8-59}
\end{array}
$$

Imagines

$$
\begin{array}{ll}
1.3 & 142 \\
2.1 & 344,35^{2}-359
\end{array}
$$

Philoxenus $6,168 \mathrm{n} 48$

Pindar 11, 23, 147, 148, 151

Olympian

$$
\begin{array}{ll}
1.1-11 & 247-248 \\
2 & 22 \\
2.1-6 & 263-264
\end{array}
$$




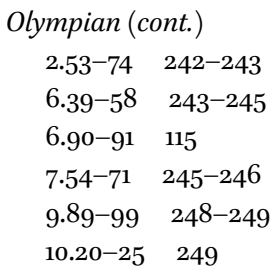

Pythian

$$
\begin{array}{ll}
1.1-4 & 265^{-266} \\
2.72 & 119-120 \\
6 & 22 \\
6.1-17 & 23 \\
7 & 22,45 \\
9 & 73-74
\end{array}
$$

Nemean

$$
\begin{array}{ll}
5 \cdot 1-5 & 23 \\
8.13^{-15} & 179
\end{array}
$$

Isthmian

$$
\begin{array}{ll}
2 & 22 \\
2.1-16 & 264-265 \\
2.43-46 & 23 \\
8.49-50 & 58
\end{array}
$$

Paean

$$
\begin{array}{lr}
2 & 267 \\
2.97 & -102 \\
4.21-24 \\
6 & 268 \\
8 & 22 \\
9 & 233
\end{array}
$$$$
\text { 2.97-102 269 }
$$$$
\text { 4.21-24 } 267
$$

fr. $94 \mathrm{~b} \quad 262-263$

fr. $122 \quad 153,155^{-157}$

fr. $123 \quad 289-290,299$

fr. 124d 158

fr. $125 \quad 159,169$

fr. $126 \quad 159$

fr. $128 \quad 158,267$

fr. 128c $\quad 267$

Plato $7,228 \mathrm{n} 42,328$

Charmides

155c-d 185

Cratylus 420a-b 303

Gorgias

$$
451 \mathrm{e} \quad 162
$$

Laws

$654 \mathrm{~b} \quad 361$ 709e $105^{n} 48$

Meno

$$
\text { 76c-d 288nı }
$$

Phaedrus

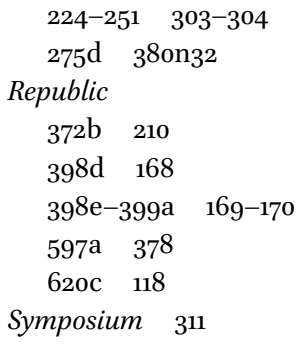

Proclus 51, 118, 268n 40

Pythermos 165,168

Quintus of Smyrna

$$
7.100 \quad 5^{8}
$$

pódov $\quad 245^{-246}, 375,378$

Romanticism 343

Sappho

fr. 1328,331

fr. $2 \quad 350-35^{2}, 362-363$ 

fr. $16295,310,319-320,326-327$
fr. 30201
fr. $31 \quad 297,326$
fr. $44 \quad 347-35^{\circ}$
fr. $58 \quad 364-366$
fr. $94 \quad 344-347$
fr. 110200
fr. 111200
fr. $112 \quad 300-301$
fr. 114200
fr. $130 \quad 300-301$
fr. 154195,316
fr. 168 b. 3 v. 316

'Brothers poem' 201

'Cologne Sappho' 305-336

sculpture $7,12,16-19,23,3^{2}, 41,45,5^{0}, 61-66$, 355,377

shield of Achilles $\quad 70-84,90,96,101$

Simonides $1,6-11,16,39,149,215,382 \mathrm{n} 40$

fr. $22.9-14 \mathrm{~W} \quad 299-300$

$P M G$

$$
\begin{array}{ll}
507 & 15^{0} \\
543 & 9 \\
55^{0} & 16 \mathrm{n} 1
\end{array}
$$

skutalê 111-115, 119, 125n64

Solon
frr. 1-3 117
fr. $4.22 \quad 121$
fr. $5.1 \quad 124$

Sophocles 42

\section{Mysians 55}

Captives 193

spectatorship $\quad 1-6,70,75^{-76}, 79,83,87,92$, $102-103,217,248,278$

symposium 109, 121-122, 135, 161-162, 170173, 186-196, 204-215, 290-291, 308, 312

synaesthesia $10,223,239,311,313,328,343$, $346,349-350,352,357,360-362$
Telestes 6

Terpander $\quad 147-148,153,159,168-169,174-180$

Theocritus 195

Idyll

18 198-199, 201

Theognis $14,115 \mathrm{n} 31,164 \mathrm{n} 35$

$467-496 \quad 210-212$

Theophrastus 123

On the Senses $\quad 288-289$

Thucydides $8,111,113$

Timocreon of Rhodes

$P M G$

$731 \quad 153,165,167$

Timotheus 6,66

tragedy $2,4,17,44,51,55^{-5} 5,61,66,151,218-$ 220, 276

transmission $\quad 192-195,307-310,362 \mathrm{n} 42$

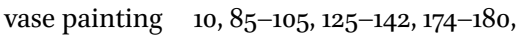
187-190, 314, 322, 328, 366

virtual chorus 311,314

vision $\quad 2-3,218,238,288,295,313,379$

visualization $2-4,86,123,128,204,217-234$, $307,313-314,322,336,343,346-361,366$

weaving $22,71,89,229,331$

wedding songs $13,70,83,148,197-201,274-$ $275,309 n 8$

Xenophanes

fr. $1 \quad 207-210$

Xenophon

Hiero 9 n2o

Memorabilia

3.11.10 185

Symposium

$2.22 \quad 122$

$4.21 \quad 381 n 34$ 